BLS 34, No 1 2008. DOI: http://dx.doi.org/10.3765/bls.v34i1.3572

(published by the Berkeley Linguistics Society and the Linguistic Society of America)

\title{
Supplemental Relative Clauses and Syntactic Generality
}

\author{
RUSSELL LEE-GOLDMAN \\ University of California, Berkeley
}

\section{Introduction}

It is generally assumed in research on non-restrictive relative structures (Ross 1984, Espinal 1991, Potts 2002a, Arnold 2007) that sentences like those in (1) are ungrammatical due to a general constraint of the form "nonrestrictive relative clauses must ... follow their antecedents" (Espinal 1991:752). The presence of such a linearization constraint seems reasonable enough given the fact that restrictive relative clauses also cannot left-adjoin (*a who I met child).

a. * Which I said earlier, there are some minor problems

b. * Who plays the piano, my father(,) knows a thing or two about music.

The reasonableness stems from the intuition that modifiers of the same class (relative clause) will be linearized similarly with respect to their heads. However appealing, this intuition is misguided, at least with respect to relative clauses. I argue that linearization of relative clauses and their heads is determined by the category of the modified head as well as that of the modifier. Further, despite indications to the contrary, non-restrictive relative clauses can left-adjoin, though the possibility for it is severely restricted (Sections 3 and 4).

The left-adjunction restriction is one of several differences adduced by Potts (2002b, 2002a) in favor of analyzing parenthetical as-clauses (As you know, this is difficult) as PPs despite their superficial similarity to which-relatives. With the left-adjunction restriction removed, however, there is a question of what differences remain that prevent bringing as into the fold of relativizers. In Section 5 I examine the putative evidence against analyzing as (in this usage) as a relative pro-sentence, finding that none of arguments presented bear on a relativizer/ non-relativizer analysis. In fact, we will see that it is as-clauses which have utterly predictable properties and which-relatives that require special pleading. Ultimately, it will be seen that linearization possibilities of a relative clause become more restricted only when information about what the relative clause adjoins to is specified. That is, the category "relative clause" defines more the internal structure of a particular type of clause, saying rather little about its external distribution. 


\section{Russell Lee-Goldman}

\section{Terminological Preliminaries}

I follow Huddleston and Pullum (2002; CGEL) in splitting relative constructions into integrated and supplemental variants, rather than restrictive and non-restrictive. For relative clauses the difference is purely terminological-supplemental relatives are non-restrictive, and integrated relatives are restrictive. But the relabeling draws a useful parallel between supplemental relative clauses and other supplements, such as parenthetical sentential adverbs (unfortunately, frankly). Rather than a modifying a head, a supplement is said to have an anchor: that part of the main clause which the supplement is semantically connected to. In the simple case of nominal apposition (the perpetrator, Jones, is right over there) an identificational relation is set up between the anchor, the perpetrator, and the supplement, Jones. I also follow Jackendoff (1977), Sag (1997), and Arnold (2007) in treating relativizers in supplemental clauses as pronominal, coindexed with their antecedent, parallel to personal pronouns (though the reference resolution process is more tightly constrained for relative pronouns). Throughout the paper I avail myself of the term antecedent (of a relativizer) to indicate that noun, clause, etc., which is modified by a particular relative clause.

\section{Supplemental Relative Clauses}

Adnominal relative clauses are subject to the same linearization constraints whether they are integrated or supplemental:

a. Kim, who I went to school with, is now a doctor.

b. The boy who I went to school with is now a doctor

a. * Who I went to school with, $\operatorname{Kim}($,$) is now a doctor.$

b. * (the) Who I went to school with (the) boy is now a doctor.

In the (2a) the supplement follows the anchor, and in (2b) the modifier follows the head; reversing the order $(3 a, b)$ is ungrammatical. Though anchors and heads are distinct grammatical functions, presumably we are dealing with the same or at least very similar constraints in both cases. At first blush supplemental relative clauses with verbal (4a) or clausal (4b) anchors follow this linearization pattern as well. For present purposes I ignore any differences between the two, and I use the term sentential for both. (5) illustrates the ungrammaticality of reversing the order.

a. You can get it from the library if you care to request it, which I have.

b. The book is twenty years old, which I discovered when I got there.

a. * Which I have, you can request the book.

b. * Which I discovered when I got there, the book is twenty years old.

Integrated and supplemental relative clauses have identical internal syntactic properties, project structures of the same category (clause), and hold similar functions (modifier and supplement, respectively). This constitutes a good motivation for treating their external syntax as identical, with a single principle of 


\section{Supplemental Relative Clauses and Syntactic Generality}

relativizer linearization. Though tempting, this would not be the right approach. Before revealing the crucial data for relative clauses, it is helpful to consider the heterogeneity of adverbs. Pullum and Huddleston (2002a), following Jackendoff (1972) split adverbs into two broad categories, clause-oriented and verb-oriented. Within each of these categories there are several types of adverbs (manner, purpose, speech-act, epistemic, etc.), and each type is associated with a set of linearization possibilities and tendencies. It should then come as no surprise that some relative clauses must be ordered in one way with respect to their heads, and others, with different types of heads, ordered in another way entirely.

\section{Left-Adjunction of Which}

In certain very restricted environments a supplemental which relative clause may appear to the left of its anchor (relative clauses are italicized, and anchors are in boldface; unless otherwise noted, sentences are from the British National Corpus):

a. Their apparently similar, sharply segmented body plan either arose more than once or-which is also more than possible - it is very primitive. ${ }^{1}$

b. Independent companies allow their directors to do away entirely with actors, and (which is the only sensible way to manufacture movies at all) pick types and faces off the streets."

c. Either they were performing this public duty in giving the protection asked for ... or, which no one suggests, they were at the request of an individual doing something which it was not their duty to do....

The existence of left-adjoined relative clauses (LARCs) is noted briefly in the CGEL (ch 13): "[a] supplementary relative with a coordinated clause as antecedent can precede it, following the coordinator" (p.1066). Breaking this down, it means that in order to license a LARC, (ia) the antecedent must be a clause, (ib) the relative clause must have the role Supplement (not Modifier), (iia) the relative clause must directly follow a conjuction, and (iib) the relative clause must have a conjunct as an antecedent.

The first two constraints are not independent of each other: (ia) entails (ib). There are no integrated relative clauses with clausal antecedents. Given that, all that need be stated is that which-clauses may have as anchors state-of-affairs- or proposition-denoting phrases. The only syntactic category that suffices for this is the clause. This is exactly parallel to constraining, e.g. who to pick out people, thus constraining it to have a nominal head. Again, these are necessary conditions for grammaticality. Even introducing conjunction in the proper configuration will not save LARCs with non-clausal antecedents: *You could talk to Sandy, or, who might also be willing to help you, Mitchell.

The supplement-only constraint on sentential relatives is not limited to

\footnotetext{
${ }^{1}$ Tudge, Colin. 2002. The Variety of Life. Oxford: Oxford University Press, p. 200.
} 


\section{Russell Lee-Goldman}

which - as the next sections show, other relativizers enter into anaphoric relations with clauses, and they too must be supplements. This generalization can be stated by arranging relativizers into a hierarchy, and state at the level of "sentential relative clause" (a constructional level) that they must appear in a structure with the role Supplement. ${ }^{2}$ Such a constraint is parallel to the restriction on relativizer that against appearing in supplemental relative clauses (Hudson 1991, Sag 1997). We thus see that a simple combination of restrictions on words and phrases yields the first two constraints on LARCs. These types of restrictions are seen elsewhere in the grammar. The only difference here is that they are relevant for linearization.

The latter two constraints, regarding conjunction, are also related: the LARC must follow a conjunction word, and what follows (its anchor) must be a conjunct:

a. * It either arose twice, which is also more than possible, or it is very primitive. [relative precedes conjunction]

b. * It may have arisen twice, which is also possible, arisen three times, or simply be very primitive. [no conjunction]

There do exist in English constructions that call for conjunctions. Several elliptical constructions, including right-node raising and gapping, require the phrases containing elided material to be conjuncts. Some lexical items might be argued to select for conjunction words: either/neither are followed by or/nor, and predicates of obligation such as must and have (got) to interact idiosyncratically with or-clauses: in I gotta leave or (else) my dad will have a fit, the or-conjunct expresses the negative consequences of not fulfilling the obligation, rather than an alternative (or additional) possibility. This, however, is lexical selection of a coordinate (or subordinate) clause. It would be inappropriate to characterize a relative clause as "selecting" a conjunction in this manner.

A flexible enough grammar might be able to constrain a LARC to appear following a conjunction, but there is a possibility that it need not be so heavy-handed: there may be semantic or pragmatic properties of left-adjoined relative clauses that make their post-conjunction position extremely likely. In particular, if one considers the entire range of left-adjoined which relative clauses that appears in the BNC, a clear semantic pattern emerges: most of the which LARCs involve a notion of scalar or nonscalar comparison of, or alternation between, two states of affairs. Most (11 of 14) are much like (6a): they include comparative phrases, nearly all as the main predicator, and nearly all preceded by or rather than and. Even of those predicates without overt comparison, one involves negation (which no one suggests) and one contains surprise (which had surprised me in the last few years), both of which are evocative of the alternative (non-surprising, non-negated) states of affairs which were mentioned in the previous conjunct.

\footnotetext{
${ }^{2}$ The relativizer is not the head of the relative clause (Sag 1997 and others), and so in a head-driven grammar, some special mechanism would be necessary if instead one wished to place the supplemental-only restriction on the relativizer, rather than the clause it appears in.
} 


\section{Supplemental Relative Clauses and Syntactic Generality}

Clearly something else is going on besides a simple linearization restriction. Space limitations preclude full development of this idea, ${ }^{3}$ but one hint that semantic or pragmatic forces are crucially involved comes from (8), which does involve a comparison: it is embedded in a context in which the antecedent is framed as personal experience, in contrast to the lengthy, scientific explanation of "crashing" from Ritalin which preceded. Despite not occurring in the environment of conjunction, it does follow the alternative/contrast-expressing tendency of LARCs, and seems to me perfectly acceptable (with the caveat that the linguistic background of online writers is hard to confirm).

Now it seems that (which I know from experience) when 'the crash' begins within 2 hours, this is when the plasma concentration ..."

This section has demonstrated the possibility for left-adjunction of relative clauses. Such a structure was shown to have several constraints, including most unusually obligatory post-conjunction position. An argument was made that some or all of these constraints are the result of a semantic/pragmatic restriction on LARCs. The next section shows that not all LARCs require which-some allow relativizer what.

\section{4. $\quad$ Left-Adjunction of What}

What is not a relativizer in standard varieties of English, but it does appear in what appear to be LARCs. Representative examples are shown in (9).

a. In other words, at these two points the crystal is pretty well broken away. What is even more important, the dislocation turns out to be movable.

b. Near-empty streets ... were no longer silent, but filled with curious brawls, or outbursts of squealing or, what he had just heard, the sound of terrified retching.

c. The segregation of people and vehicles, what is more, has led to new and unforeseen problems.

Aside from its peculiar linearization, these clauses act just like canonical relative clauses, with a clause-initial relativizer in a long-distance dependency with a gap in the clause: What I \{am sure he considers / *met someone who considers\} even more important, the dislocation turns out to be movable.

\footnotetext{
${ }^{3}$ One promising possibility may comes from considering LARCs to be complex discourse connectives, and thus evocative of the types of discourse coherence proposed by Kehler (2000) and Asher and Lascarides (2003). The relations parallel, contrast, and alternative seem most relevant, and indeed most of these involve conjunction. See Kehler (2000) for indications that comparison falls into the same (resemblance-based) class as parallel and contrast.

${ }^{4} \mathrm{http}: / / \mathrm{mb}$. rxlist.com/rxboard/ritalin.pl?noframes;read=526, accessed 2008.05.01
} 


\section{Russell Lee-Goldman}

Of the 32 examples extracted from the $\mathrm{BNC},{ }^{5} 25$ are subject relatives with comparative predicators (e.g. (9a)) and four involve same (e.g. what amounts to the same thing). Comparison is clearly an integral part, just as for which. In fact, removing indications of comparison (more, also) results in unacceptability: Someone who climbed over a fence instead of using the official entrance (or, what is *(more/also) likely, exit) was deemed disorderly.

In general, then, the same constraints seen on which-LARCs hold as well for what, with a few exceptions: Although what-LARCs often follow conjunctions, they need not (10a). The antecedent is also almost always clausal, but sometimes it is not (10b). ${ }^{6}$ This points to what-LARCs following a tendency similar but not identical to that of which. What exactly that difference is (and it seems likely to be pragmatic rather than syntactic) is left as a mystery.

Finally, there is an idiomatic form, what be more, which has a much wider distribution - in fact, essentially the same distribution described in the CGEL for sentential supplemental adverbs (frankly, surprisingly), what Ross (1984) described as being "niched":

(10) a. [W]e can hardly fail to notice the sympathy ... a sympathy, what is more, that nowadays would often be slapped down as a sentimental, modern, postwar "permissive" fad.

b. How had that fancily-named high-hab brat come to be here at the garrison block? Mingling, what's more, with Dorcas gang members, or so it seemed?

c. I was rude," he declared, "and very unreasonable, what's more.

What this demonstrates is that the label "LARC" is actually a bit misleading when applied to what be more. To the extent that this phrase has the normal distribution for a sentence-level supplement, it is not particularly notable that it can appear to the left or right of anything, let alone some constituent that it appears to modify. In fact it is more appropriate to say that because it modifies the sentence, it may appear anywhere within that sentence, subject only to the general constraints on supplements. This is a crucial generalization, one which will be returned to in discussion of as. The non-idiomatic versions of what-relatives, on the other hand, may be more appropriately be called LARCs. A relative clause like what is more interesting is importantly limited to either post-conjunction or sentence-initial position (except, of course, as a free relative, in which case its distribution closely

\footnotetext{
${ }^{5}$ Only punctuation-preceded tokens have been extracted. The 32 examples do not include the form what be more, of which there are 57 tokens.

${ }^{6}$ In some cases it seems as though which has a nominal antecedent: The starting point can be either a known position such as a radio beacon over which the aircraft has flown or, which is more likely in the case of an accident, the crash position. The anchor here is the NP the crash position, but I argue that here we are dealing with a type of metonymy, in which case the antecedent is in fact some state-of-affairs in which the crash position holds a role, namely "being the starting point."
} 


\section{Supplemental Relative Clauses and Syntactic Generality}

matches that of NPs). In either case, what it is modifying (and more on this below) follows it, and so we may speak of a "left-adjunction" as a property holding of the construction.

\section{Supplemental Relativizer $A s$}

Just as was the case for which, supplemental as-clauses may be grouped into two types: those with a predicate gap and those with a clause gap (again I use sentential-as as a cover term.

a. Art is not, as the metaphysicians say _, the manifestation of some Idea of beauty or God

b. There are a number of options available to the publisher who is stuck, as most of us are , with the A4 page.

a'. * Art is not, as I met a metaphysician who said , the manifestation...

b'. * He was stuck with the A4 page, as he had read a manual that told him publishers often are

The similarities with relative clauses include: a "functional" word preceding a finite gapped clause, the gap being either predicative or clausal, corresponding to modification of a predicative or clausal or antecedent. The gap is, further, in a long-distance dependency with something outside the finite clause and thus island effects can be observed (11a',b'; see Potts (2002a,b) and citations within for extensive discussion). Finally, the semantic content of that gap is filled by main-clause material adjacent to the as-clause: in (11a) what metaphysicians say is that "art is not the manifestation of...God," and what "most of us are" in (11b) is "stuck with the A4 page.

The as-clause, as I will call any gapped finite clause with an initial as, has several properties of a relative clause, especially supplemental which relatives. This section argues that treating as as a relativizer is indeed the preferred approach. This is by no means a new claim - it is in fact the traditional analysis (see the OED's entry), assumed by Kayne (1984) and Postal (1994, 2004), and hinted at by Ross (1984) - but it has recently been argued against by Potts (2002a,b) and the CGEL (chapter 13). Here my purpose is not to present particular arguments in favor of relativization, but rather to defuse the variety of putative arguments against it (see Lee-Goldman in prep. for positive arguments).

Potts (2002a) gives two reasons for not treating as as the extracted element, favoring a null operator approach. He posits two as lexical items, one for predicate-modifying uses and one for clause-modifying uses. Their denotations and location in the tree proposed are given in 12 and 13. As is a preposition that selects a CP with a phonologically-null operator merged at the cite of the gap. Semantic composition at the level at which the PP attaches to the anchor guarantees that the anchor's semantics will fill in the missing material in the as-clause. This is opposed to the relativizer account proposed here, in which the as itself enters into a long-distance dependency with the gap, and thus the as-clause is a clause rather 


\section{Russell Lee-Goldman}

than a PP and semantic composition is handled by coindexation (Sag 1997).
a. $[[\mathrm{CP}-a s]]=\lambda \mathrm{Q} \in \mathrm{D}_{<<\mathrm{st}, \mathrm{t}, \mathrm{t}>}\left[\lambda \mathrm{p} \in \mathrm{D}_{<\mathrm{st} \triangleright}: \mathrm{Q}(\mathrm{p})\right.$ is true $\left.[\mathrm{p}]\right]$
b. [anchor Dan swims] [pp as [CP OP C you know $<\mathrm{OP}>$ ]]
a. $\quad[[$ Pred- $a s]]=\lambda \mathrm{F} \in \mathrm{D}_{<<\mathrm{s}, \mathrm{et}>, \mathrm{t}}\left[\lambda \mathrm{f} \in \mathrm{D}_{<\mathrm{s}, \mathrm{et}}: \mathrm{F}(\mathrm{f})\right.$ is true $\left.[\mathrm{f}]\right]$
b. Dan [anchor Swims] [PP as [CP OP C my sister used to $<\mathrm{OP}>$ ]]

\subsection{Two Non-Arguments}

Potts (2002b) presents two arguments against relativization. The first is that as is blocked from appearing in its "base" position, unlike other pro-sentential words like so: I hoped so and so I hoped are both fine, but as we all hoped is paired with *we all hoped as. Though this is true, it does not thus rule out a relativizer analysis, as relative pronouns do not ever appear in situ (*the child you met whom). Even if one counts wh-in-situ question words as somehow fulfilling this extracted/in situ alternation test, there is still relativizer that and the question word how come, which never appear in their "base" position, though they are almost surely extracted elements. The non-occurrence of post-verbal as is thus equally well predicted under both styles of analysis. ${ }^{7}$

Potts's second argument comes from cross-linguistic comparison of as-like constructions. He finds that the analogous word in Thai is yaan, which is derived from the question word for 'how.' This is illustrated in (14), from Potts's (2002b) sentence (35):

$$
\begin{aligned}
& \mathrm{t}^{\mathrm{h}} \mathrm{u} \text { ?.rian Pa.roy yaan } \mathrm{t}^{\mathrm{h}} \mathrm{ii} \mathrm{k}^{\mathrm{h}} \text { on ee. } \mathrm{c}^{\mathrm{h}} \text { ian laai } \mathrm{k}^{\mathrm{h}} \text { on ruu } \\
& \text { durians delicious as C Asian many class know } \\
& \text { 'Durians are delicious, as many Asians know.' }
\end{aligned}
$$

The argument goes as follows. The problem with analyzing yaan as extracted is that Thai is a wh-in-situ language, and so one would be required to posit for this particular construction an exception to the rule that operators may not be extracted. This problem does not arise if yaan is a preposition. A severe problem exists with this solution, however, and that is the fact that the subordinate clause still contains a gap, even in Thai, which is still unexpected for a wh-in-situ language. This must be accomplished somehow, presumably by a phonologically-null constituent that moves to a higher position during the derivation. What this does, however, is require whatever it is that prevents operators from undergoing extraction to recognize when a particular lexical item will be phonologically exponed and block movement. Or, if one says that this particular operator does not move until semantic interpretation occurs, then one must somehow constrain the operator to appearing in yaan-clauses. Either of these might be accomplished with some sort of diacritic

\footnotetext{
${ }^{7}$ The impossibility of pied-piping with as is similarly unproblematic under a relativization analysis, as pied-piping already requires a particular type of relative pronoun (Hudson 1991), and as may simply not be in that category.
} 


\section{Supplemental Relative Clauses and Syntactic Generality}

on the operator, but the facts could just as well be accounted for with a special diacritic allowing movement. The Thai facts do not seem to come down on one side or the other.

\subsection{Some Inconsequential Differences}

Potts (2002a), building on observations by Ross (1984) and Postal (1998), picks out five differences between which and as: (1) As-clause gaps are non-nominal, while which-clause gaps are nominal, (2) only as-clauses may be "niched," (3) only as-clause gaps are sensitive to negative and wh-islands, (4) only which-clause gaps may be the subjects of "CP-equatives," and (5) as-clauses may, depending on their position, "ignore" main-clause negation and modality when determining the semantic content of their gap; which-clauses may not. Two further differences are cited by Pullum and Huddleston (2002b): (6) as-clauses, but not which-clauses, present backgrounded information, and (7) as-clauses have several properties of comparatives not present in which relative clauses, including the possibility for subject-auxiliary inversion and the various types of reduction associated with comparative clauses.

A full treatment of all of these differences is given in Lee-Goldman (in prep). Most of these are not syntactic but semantic or pragmatic: Potts (2002a) argues this for (3) and (4); (6) is already a pragmatic difference, and (5) is related to niching, Here I concentrate on the (non)-nominal nature of the gap, niching restrictions, and comparative properties of as, determining ultimately that these differences do not impact the relativizer/non-relativizer analysis.

At least as far back as Stowell (1987) (which I have not seen), but also in Postal (1994) and Potts (2002b), it has been observed that the gaps in as-clauses are not nominal, while those in sentential which relative clauses are. One piece of evidence (Potts goes through several) is that aware, which may take either a clausal or prepositional complement, shows different valences with as and which:

(15) a. He is doing fine, as I'm sure you're aware (*of).

b. He is doing fine, which I'm sure you're aware *(of).

Because of is incompatible with the non-nominal valence (*aware of that he was okay), it is incompatible with as-clauses.

This first difference between the two words does not have any bearing on the relativizer/non-relativizer question. There is no a priori reason to exclude a non-nominal relativizer from the lexicon (just as there would be no reason to exclude whatever variety of null operators must be posited for the structures in (12) and (13)). The nominal/non-nominal distinction is an important one, however, and will figure in the discussion of linearization.

The first few sections of this paper showed that linearization with respect to relative clauses and their antecedents is anything but cut and dried. The exact ordering possibilities depend on the type of relativizer in addition to the syntactic (and possibly pragmatic) category of the anchor and antecedent. Nevertheless, one 


\section{Russell Lee-Goldman}

might be concerned at the sheer number of possible locations that as-clauses can appear in - is it not highly unusual for a relative clause? A schematic indication of where it may appear is in (16). In each place indicated with a " " a clause like as I said is possible. A subscripted "*”, "?", etc., indicate varying degrees of acceptability (according to my own intuitions).

$$
\wedge \mathrm{He} \wedge \text { has } \wedge \text { been } \wedge \text { too ? willing } \text { to } \wedge \text { help ? students } * \text { of } * \text { literature } \wedge
$$

Ross (1984) noted this possibility for niching and characterized the linearization possibilities as "roughly the same ... as any other sentence adverb would" (p.261). The question is then whether we can reasonably expect as-clauses to have such a range of linearization possibilities, and I think the answer must be that we can. As we have seen, the real set of linearization criteria includes the syntactic category of both the modifier and the anchor/head, so it should come as no surprise that as-clauses, as modifiers of clauses, can appear exactly where other, lexical, modifiers of such structures do. Linearization is not a "problem" for a relativizer analysis, because the determination that a particular structure is a relative clause reveals little about its linearization possibilities. It is only when we learn that it is supplemental or integrated, and further whether it is adnominal or sentential that the linear ordering becomes more fixed.

From this perspective, what requires investigation is the comparatively limited distribution of supplemental which-clauses, which appear never to interrupt their anchors. Given that they modify sentences just as much as as-clauses do, should they not have an equally flexible distribution?

The answer to this question may lie in the observation made by Postal (1994) and later reinforced by Potts (2002a) that gaps in which-clauses are truly nominal. Potts (2002a) took this one step further and demonstrated that clause's anchor (i.e. the main clause) must also be nominalized. It then becomes very relevant that adnominal relative clauses must follow their anchors, while sentential which supplements often follow, but at least must be adjacent to their anchors. Though this is tentative, there is some sense in which the fact that their pre-anchor position is so pragmatically limited indicates that which clauses are still holding on to (or have a special affinity with) their adnominal roots: they must be adjacent to their anchors. The flexibility they do have is perhaps the beginning of a shift to a wider distribution more in accordance with their status as sentential modifiers.

Such retention of vestigial constructional features is not limited to which clauses. As noted above, supplemental as-clauses with predicative gaps allow subject-auxiliary inversion (as have I). This too is likely a "vestigial" feature, due to affinity with historically related but now separate constructions. Further examples of this sort of vestigial feature can be found in other places in the grammar, for instance non-inversion in how-come questions, quasi-auxiliary properties of dare and better, and complementizer-like properties of relativizer that.

A final argument for treating linearization as non-diagnostic of relativizer status 


\section{Supplemental Relative Clauses and Syntactic Generality}

comes from another supplemental use of as, what I will call Name-as. It too precedes a finite clause with a gap, but in this case the gap is a "name" of some sort.

(17) a. The best thing is my ability to "vague out", as I call it

b. This is the account I attribute to, as I call him, 'Sartre-Two'.

c. * Trish has been working too hard, as I call her for short.

If linearization is determined by what sort of clause the modifier is, then we should expect Name-as clauses to have the same distribution as sentential as-clauses. This is predicted by the CGEL, which attributes the niching properties of as to the fact that is a self-selecting, or ungoverned adjunct (p.1147). Name-as is ungoverned in their sense, yet it is just as limited as which and what: it may appear following the name it modifies (17a), or preceding it (17b), but nowhere else. The reason for this is of course that as a modifier of names, Name-as must appear local to that modified head. To reiterate, the distribution of a relative clause is not determined strictly by the category of the modifier; what it modifies matters as well. Sentential as has more liberty to move around because it simply has to appear within the sentence it modifies, like any well-behaved sentential supplement.

\section{Conclusion}

Table 1 summarizes the properties of the constructions examined so far. Each row indicates for a given construction whether it may appear after its anchor, before it, and whether it can be niched.

Table 1: Relative Clause Linearization Possibilities

\begin{tabular}{|c|l|l|l|}
\hline clause type & \multicolumn{1}{|c|}{ Post-anchor } & \multicolumn{1}{|c|}{ Pre-anchor } & \multicolumn{1}{|c|}{ Niching } \\
\hline Which & Yes & Limited $^{\mathrm{a}}$ & No \\
\hline What & Yes & Yes & Limited $^{\mathrm{b}}$ \\
\hline Adnominal & Yes & No & No \\
\hline Name- $a s$ & Yes & Yes & No \\
\hline Sentential- $a s$ & Yes & Yes & Yes \\
\hline Sentential adverb & Yes & Yes & Yes \\
\hline
\end{tabular}

Only following a conjunction

${ }^{\mathrm{b}}$ Only when in the idiomatic form what be more

What this reveals is tremendous diversity among relative clauses. Adnominal relative clauses have all the expected properties, but interestingly no other construction in the table has exactly those properties. Both which and what are more flexible, though with some strong constraints on that flexibility. As relatives also vary according to their anchor's category, with sentential as patterning with sentential adverbs. What this shows is that, even among a class unified by their

\footnotetext{
${ }^{8}$ Young, Julian. 2003. The Death of God and the Meaning of Life. London: Routledge, p. 142
} 


\section{Russell Lee-Goldman}

internal syntactic properties, individual members may have rather different external syntactic (linearization) properties.

\section{References}

Arnold, Doug. 2007. Non-Restrictive Relatives Are Not Orphans. Journal of Linguistics 43:271-309.

Espinal, Maria Teresa. 1991. The Representation of Disjunct Constituents. Language 67:726-762.

Huddleston, Rodney, and Geoffrey K. Pullum. 2002. The Cambridge Grammar of the English Language. Cambridge: Cambridge University Press.

Hudson, Richard. A. 1991. English Word Grammar. Oxford: Blackwell.

Jackendoff, Ray. 1972. Semantic Interpretation in Generative Grammar. Cambridge: MIT Press.

Jackendoff, Ray. 1977. X-bar Syntax. Cambridge: MIT Press.

Kayne, Richard. S. 1984. Connectedness and Binary Branching. Dordrecht: Foris Publishers.

Lee-Goldman, Russell. In preparation. The Unity and Diversity of Supplemental Sentential Relative Clauses. Ms. University of California, Berkeley.

Postal, Paul. M. 1994. Parasitic and Pseudo-Parasitic Gaps. Linguistic Inquiry 25:63-117.

Postal, Paul. M. 1998. Three Investigations of Extraction. Cambridge, Massachusetts: The MIT Press.

Postal, P. M. 2004. A Paradox in English Syntax. In Skeptical Linguistics Essays, 15-82. Oxford: Oxford University Press.

Potts, Christopher. 2002a. The Lexical Semantics of Parenthetical-As and Appositive-Which. Syntax 5:55-88.

Potts, Christopher. 2002b. The Syntax and Semantics of As-Parentheticals. Natural Language and Linguistic Theory 20:623-689.

Ross, John R. 1984. Inner Islands. In C. Brugman \& M. Macaulay, eds., Proceedings of the 10th Annual Meeting of the Berkeley Linguistics Society, 258-273. Berkeley: Berkeley Linguistics Society.

Sag, Ivan. A. 1997. English Relative Clause Constructions. Journal of Linguistics 33:431-483.

Russell Lee-Goldman

University of California, Berkeley

Department of Linguistics

1203 Dwinelle Hall

Berkeley, CA 94720-2650

rleegold@berkeley.edu 